\title{
EFFICIENCY AND GENERALISED CONVEXITY IN VECTOR OPTIMISATION PROBLEMS
}

\author{
PHAM HUU SACH ${ }^{1}$, GUE MYUNG LEE ${ }^{2}$ and DO SANG KIM ${ }^{2}$
}

(Received 11 September, 2001; revised 10 January, 2002)

\begin{abstract}
This paper gives a necessary and sufficient condition for a Kuhn-Tucker point of a nonsmooth vector optimisation problem subject to inequality and equality constraints to be an efficient solution. The main tool we use is an alternative theorem which is quite different to a corresponding result by $\mathrm{Xu}$.
\end{abstract}

\section{Introduction}

A vector optimisation problem is a problem where two or more objectives are to be minimised on some set of feasible solutions. In such a problem we often deal with conflicts amongst objectives and hence in most cases cannot find a feasible solution which is optimal in the sense that it minimises all the objectives simultaneously. So in vector optimisation we must use concepts different from this requirement of optimality. In this paper, we restrict ourselves to the concept of an efficient solution: this is a feasible solution such that there does not exist another feasible solution at which all objectives are the same or better, with at least one being strictly better. From a mathematical viewpoint it can be formulated as follows. Let us consider a set $S_{1}$ of an Euclidean space $\mathbb{R}^{n}$ and $m$ functions $f_{i}(i=1,2, \ldots, m)$ defined on $\mathbb{R}^{n}$. The set $S_{1}$ can be interpreted as the set of feasible solutions and the functions $f_{i}$ can be regarded as our objectives which we want to minimise. Then a point $x_{0} \in S_{1}$ is an efficient solution if we cannot find another point $x \in S_{1}$ such that $f_{i}(x) \leqq f_{i}\left(x_{0}\right)$ for all $i$ and, in addition, at least one of these inequalities is strict. This efficiency property originated with Pareto [16] and plays a crucial role in economics, game theory and statistical decision theory (see $[1,3,7,16,22,24])$. In many practical situations $S_{1}$ is

\footnotetext{
${ }^{1}$ Hanoi Institute of Mathematics, P.O. Box 631, Boho, Hanoi, Vietnam; e-mail: phsach@math.ac.vn.

${ }^{2}$ Department of Applied Mathematics, Pukyong National University, Pusan 608-737, Korea; e-mail: gmlee@pknu.ac.kr and dskim@pknu.ac.kr.

(C) Australian Mathematical Society 2004, Serial-fee code 1446-1811/04
} 
given as a subset of a closed set $S$ which consists of all points $x$ of $S$ satisfying a system of inequalities and equalities:

$$
g_{j}(x) \leqq 0(j=1,2, \ldots, k), \quad h_{s}(x)=0(s=1,2, \ldots, l) .
$$

In addition, the functions $f_{i}, g_{j}$ and $h_{s}$ are not differentiable in the classical sense. In this paper they are assumed to be locally Lipschitz. Such functions are often encountered in economics, engineering design and various branches of analysis. Examples of locally Lipschitz functions arising in these fields can be found in [5] which is a basic book for everyone interested in nonsmooth analysis. The problem of finding efficient solutions for the objectives $f_{i}$ on the above feasible set $S_{1}$ is referred to as problem $(V O P)$ and is written as follows:

$$
\text { Minimise } \begin{aligned}
f(x) & :=\left(f_{1}(x), f_{2}(x), \ldots, f_{m}(x)\right) \text { subject to } \\
g_{j}(x) & \leqq 0 \quad(j=1,2, \ldots, k), \\
h_{s}(x) & =0 \quad(s=1,2, \ldots, l), \\
x & \in S .
\end{aligned}
$$

It is well-known [12] that the convexity of functions involved in a minimisation problem with inequality constraints (that is, problem (VOP) where $p=1, S=\mathbb{R}^{n}$ and $h_{s}$ are absent) assures the optimality of a point satisfying the Kuhn-Tucker conditions [12] and the validity of the Wolfe duality theorems [12]. In 1981, Hanson [9] was the first to show that a generalised convexity requirement, later called invexity, is an appropriate substitute for the usual convexity condition in proving these facts. The invexity idea is also useful for establishing necessary optimality conditions $[10,11]$ and alternative theorems [4]. In [13] the invexity property was extended to KT-invexity to prove that a Kuhn-Tucker point (that is, a point satisfying the Kuhn-Tucker necessary optimality conditions) is a minimiser of a minimisation problem with differentiable data if and only if this program is KT-invex at this point. A generalisation of invexity to locally Lipschitz functions was introduced in $[6,17,18]$. It has been noted [21] that invexity is not suitable for problems with equality constraints since the Kuhn-Tucker multipliers associated with these constraints are not necessarily nonnegative. So a new notion of infine functions was defined and was shown in [21] to be an adequate tool for equality constraints. Observe from [21, Remark 3.5] (see also Remark 4.2 of the present paper) that introducing a new terminology for infineness is needed since the class of locally Lipschitz infine functions does not coincide with the class of cone-invex functions defined by Craven [6]. The invex-infineness property (that is, the requirement of invexity for objectives and inequality constraints, and of infineness for equality constraints) is used in [21] to establish a necessary and sufficient condition for an efficient solution to be a Geoffrion properly efficient solution [8] in problem (VOP) with locally Lipschitz data. 
The aim of this paper is to extend the invex-infineness to KT-pseudoinvex-infineness and GKT-pseudoinvex-infineness such that under suitable assumptions a Kuhn-Tucker point $x_{0}$ is an efficient solution of (VOP) if and only if (VOP) is KT-pseudoinvex-infine (or GKT-pseudoinvex-infine) at $x_{0}$. Roughly speaking, we want to generalise a known result of Martin [13, Theorem 2.1] to the case of efficient solutions of nonsmooth multiobjective problems subject to mixed constraints (1.1)-(1.3). The proof of the above result and other related facts in Section 4 is based on an alternative theorem which is established in Section 3 for a system of inequalities and equalities given by the support function of nonempty convex compact sets. When each of these sets is a singleton we obtain a result (Corollary 3.2) which is quite different from the nonhomogeneous Farkas lemma of $\mathrm{Xu}$ [23]: $\mathrm{Xu}$ restricts himself to the case when equalities are absent and some additional hypothesis is required for the validity of his Farkas lemma while our Corollary 3.2 is true without these restrictions. (The formulation of Xu's result and that of our own is also different.) Section 3 also contains an application of our alternative result to a concave vector optimisation problem subject to several concave inequality constraints and abstract linear constraints. Observe that applications of concave programming problems arise more frequently in areas such as inventory, production and transportation planning, site selection, Leontiev substitution systems, assignment problems, decision theory, network flows and so forth. The reader is referred to [2] for a comprehensive survey of the theory of concave programming and an overview of its applications.

To conclude this introduction let us observe from Remark 4.4 that our paper contains results which can be applied to any practical problem with inequality constraints. This shows the applicability of some of our theoretical results to a wide class of problems often encountered in practice.

\section{Preliminaries}

Let $\mathbb{R}^{n}$ be an Euclidean space. For $x=\left(x_{1}, \ldots, x_{n}\right) \in \mathbb{R}^{n}$ and $y=\left(y_{1}, \ldots, y_{n}\right) \in$ $\mathbb{R}^{n}$ we will use the following notation:

$$
\begin{aligned}
& x=y \Leftrightarrow x_{i}=y_{i}, \quad \text { for all } i \\
& x<y \Leftrightarrow x_{i}<y_{i}, \quad \text { for all } i \\
& x \leqq y \Leftrightarrow x_{i} \leqq y_{i}, \quad \text { for all } i \\
& x \leq y \Leftrightarrow x \leqq y \text { and } x \neq y ; \\
& x \notin y: \text { the negation of } x \leq y .
\end{aligned}
$$

Let us observe that if $n=1$, that is, if $x$ and $y$ are real numbers then the above notation shows that $x \leq y \Leftrightarrow x<y$. 
If $I$ is a nonempty subset of $\{1,2, \ldots, n\}$ we will denote by $\lambda_{I}$ or $\left(\lambda_{i}\right)_{i \in I}$ the vector with components $\lambda_{i}(i \in I)$. Similarly, if $f_{i}: \mathbb{R}^{n} \rightarrow \mathbb{R}(i \in I)$ is a function then we will use the symbol $f_{l}$ or $\left(f_{i}\right)_{i \in l}$ to denote the vector-valued map with components $f_{i}$ $(i \in I)$.

Let $f: \mathbb{R}^{n} \rightarrow \mathbb{B}$ be a locally Lipschitz function, that is, for any $z \in \mathbb{R}^{n}$, there exist $\alpha>0, \beta>0$ such that for any $x, x^{\prime} \in \mathbb{R}^{n}$ with $\|x-z\|<\alpha,\left\|x^{\prime}-z\right\|<\alpha$, $\left|f(x)-f\left(x^{\prime}\right)\right| \leqq \beta\left\|x-x^{\prime}\right\|$, and let $x_{0} \in \mathbb{R}^{n}$. Then the Clarke directional derivative of $f$ at $x_{0}$ in the direction $v$ is defined by

$$
f^{0}\left(x_{0} ; v\right)=\limsup _{y \rightarrow x_{0} \lambda \downarrow 0} \frac{f(y+\lambda v)-f(y)}{\lambda}
$$

and the Clarke subdifferential of $f$ at $x_{0}$ is defined by

$$
\partial f\left(x_{0}\right)=\left\{\xi \in \mathbb{R}^{n}: f^{0}\left(x_{0} ; v\right) \geqq\langle\xi, v\rangle \dot{\forall} v \in \mathbb{R}^{n}\right\},
$$

where $\langle\cdot, \cdot\rangle$ denotes the inner product in $\mathbb{R}^{n}$.

It is well-known [5] that for any $v \in \mathbb{R}^{n}$

$$
f^{0}\left(x_{0} ; v\right)=\max _{\xi \in \partial f\left(x_{0}\right)}\langle\xi, v\rangle
$$

and $\partial f\left(x_{0}\right)$ is a nonempty compact convex subset of $\mathbb{R}^{n}$. When $f$ is of class $C^{1}$ then $\partial f\left(x_{0}\right)$ coincides with the Fréchet derivative $f_{x_{0}}^{\prime}$ of $f$ at $x_{0}$ (see [5]). If $f_{I}$ is a vector-valued map with locally Lipschitz components $f_{i}(i \in I)$ then we denote by $f_{I}^{0}\left(x_{0} ; \cdot\right)$ the vector-valued map with components $f_{i}^{0}\left(x_{0}^{*} ; \cdot\right)(i \in I)$. The symbol $f_{x_{0}}^{\prime}$ is used to denote the matrix with row vectors $f_{i x_{0}}^{\prime}(i \in I)$. Thus $f_{x_{0}}^{\prime} \eta$ is simply the vector with components $f_{i x_{0}}^{\prime} \eta:=\left\langle f_{i x_{0}}^{\prime}, \eta\right\rangle(i \in I)$.

Let $S$ be a closed subset of $\mathbb{B}^{n}$ and $x_{0} \in \mathbb{R}^{n}$. The Clarke [5] tangent cone of $S$ at $x_{0}$ is defined by

$$
T_{S}\left(x_{0}\right):=\left\{v \in \mathbb{R}^{n}: d_{s}^{0}\left(x_{0} ; v\right)=0\right\},
$$

where $d_{S}(x)=\inf _{z \in S}\|z-x\|$, and the Clarke [5] normal cone of $S$ at $x_{0}$ is defined by

$$
N_{S}\left(x_{0}\right):=\left\{w \in \mathbb{R}^{n}:\langle v, w\rangle \leqq 0 \forall v \in T_{S}\left(x_{0}\right)\right\} .
$$

A subset $A \subset \mathbb{R}^{n}$ is said to be a cone if $\lambda x \in A$ for all $x \in A$ and $\lambda \geqq 0$. A cone which is a convex set is said to be a convex cone. For any nonempty subset $A \subset \mathbb{R}^{n}$ denote by cone $A$ the intersection of all convex cones containing $A$. It is easy to check that cone $A$ is a convex cone consisting of all points of the form $\sum_{i=1}^{m} \lambda_{i} x_{i}$ where $m$ is a positive integer, $x_{i} \in A$ and $\lambda_{i} \geqq 0$. Also cone $A=\operatorname{cone}(\operatorname{co} A)$, where $\operatorname{co} A$ stands for the convex hull of $A$. When $A$ is a convex set, cone $A=\{\lambda x: \lambda \geqq 0, x \in A\}$. It has been proved [5] that $N_{S}\left(x_{0}\right)=\mathrm{cl}$ cone $\partial d_{s}\left(x_{0}\right)$, where $\operatorname{cl} A$ denotes the closure of $A$. Also we denote by $s p A$ the intersection of all subspaces of $\mathbb{R}^{n}$ containing $A$. Observe that $\operatorname{sp} A=$ cone $A-$ cone $A$. 


\section{An alternative theorem}

In this section we give an alternative theorem which is needed to prove the results of Section 4. Let

$$
\begin{array}{ll}
f_{i}(x)=\max _{v \in B_{i}}\langle v, x\rangle, & i \in I:=\{1,2, \ldots, m\}, \\
g_{j}(x)=\max _{v \in C_{j}}\langle v, x\rangle, & j \in J:=\{1,2, \ldots, k\}, \\
h_{s}(x)=\max _{v \in A_{s}}\langle v, x\rangle, & s \in L:=\{1,2, \ldots, l\},
\end{array}
$$

where $B_{i}(i \in I), C_{j}(j \in J)$ and $A_{s}(s \in L)$ are nonempty convex compact subsets of $\mathbb{R}^{n}$. Let $S$ be a closed convex cone in $\mathbb{R}^{n}$. Setting $\tilde{h}_{s}(x)=\max _{v \in-A_{s}}\langle v, x\rangle$, we see that

$$
\left.\begin{array}{l}
h_{s}(x) \leqq a_{s} \\
\tilde{h}_{s}(x) \leqq-a_{s}
\end{array}\right\} \Longrightarrow h_{s}(x)=a_{s}
$$

Let $S^{-}:=\left\{\xi \in \mathbb{R}^{n}:\langle\xi, x\rangle \leqq 0\right.$ for any $\left.x \in S\right\}$. Then we can check that $x \in S$ if and only if

$$
q(x) \leqq 0
$$

where $q(x)=\max _{\xi \in D}\langle\xi, x\rangle, D:=S^{-} \cap B^{n}$ and $B^{n}$ is the closed unit ball of $\mathbb{R}^{n}$.

The following lemma will be needed for obtaining our alternative theorem.

LEMMA 3.1 ([19,20]). The system of inequalities $f_{i}(x)<0(i \in I), g_{j}(x) \leqq 0$ $(j \in J)$ has a solution if and only if $0 \notin \operatorname{co} \bigcup_{i \in I} B_{i}+$ cl cone $\bigcup_{j \in J} C_{j}$.

Now let $a=\left(a_{1}, a_{2}, \ldots, a_{l}\right), b=\left(b_{1}, b_{2}, \ldots, b_{m}\right)$ and $c=\left(c_{1}, c_{2}, \ldots, c_{k}\right)$ and let $A_{s}^{\prime}=A_{s} \times\left\{-a_{s}\right\} \subset \mathbb{R}^{n} \times \mathbb{R}, B_{i}^{\prime}=B_{i} \times\left\{-b_{i}\right\} \subset \mathbb{R}^{n} \times \mathbb{R}$ and $C_{j}^{\prime}=C_{j} \times\left\{-c_{j}\right\} \subset \mathbb{R}^{n} \times \mathbb{R}$.

We will need the following closedness assumption $(\mathrm{H})$.

(H) For each $p \in I$ the set

$$
\operatorname{cone}\left\{\bigcup_{i \neq p} B_{i}^{\prime}, \bigcup_{j \in J} C_{j}^{\prime}\right\}+\operatorname{sp} \bigcup_{s \in L} A_{s}^{\prime}+\left[S^{-} \times\{0\}\right]
$$

is closed.

REMARK 3.1. Assumption (H) automatically holds if each of the sets $B_{i}, C_{j}$ and $A_{s}$ is a singleton and if $S^{-}$is a polyhedral cone. 
THEOREM 3.1. Assume that the closedness assumption $(\mathrm{H})$ holds. Consider the following statements:

(a) The system

$$
f(x) \leq b, \quad g(x) \leqq c, \quad h(x)=a, \quad x \in S
$$

has a solution, where $f=\left(f_{i}\right)_{i \in I}, g=\left(g_{j}\right)_{j \in J}$ and $h=\left(h_{s}\right)_{s \in L}$;

(b)

(I)

$$
\left\{\begin{array}{l}
\left(\lambda_{i}\right)_{i \in I}>0, \quad\left(\mu_{j}\right)_{j \in J} \geqq 0, \quad\left(\delta_{s}\right)_{s \in L} \geqq 0, \quad\left(\bar{\delta}_{s}\right)_{s \in L} \geqq 0, \\
0 \in \sum_{i \in I} \lambda_{i} B_{i}+\sum_{j \in J} \mu_{j} C_{j}+\sum_{s \in L} \delta_{s} A_{s}-\sum_{s \in L} \bar{\delta}_{s} A_{s}+S^{-}, \\
0=\sum_{i \in I} \lambda_{i} b_{i}+\sum_{j \in J} \mu_{j} c_{j}+\sum_{s \in L} \delta_{s} a_{s}-\sum_{s \in L} \bar{\delta}_{s} a_{s},
\end{array}\right.
$$

or

$$
\left\{\begin{array}{l}
\left(\lambda_{i}\right)_{i \in I} \geqq 0, \quad\left(\mu_{j}\right)_{j \in J} \geqq 0, \quad\left(\delta_{s}\right)_{s \in L} \geqq 0, \quad\left(\bar{\delta}_{s}\right)_{s \in L} \geqq 0, \\
0 \in \sum_{i \in I} \lambda_{i} B_{i}+\sum_{j \in J} \mu_{j} C_{j}+\sum_{s \in L} \delta_{s} A_{s}-\sum_{s \in L} \bar{\delta}_{s} A_{s}+S^{-}, \\
0>\sum_{i \in I} \lambda_{i} b_{i}+\sum_{j \in J} \mu_{j} c_{j}+\sum_{s \in L} \delta_{s} a_{s}-\sum_{s \in L} \bar{\delta}_{s} a_{s},
\end{array}\right.
$$

has a solution.

Then

(1) (a) does not hold $\Longrightarrow$ (b) holds.

(2) If we assume additionally that for any $s \in L, A_{s}$ is a singleton, then we can state that either (a) or (b) holds, but never both.

Proof. (1) Suppose that (a) does not hold. Then system (3.3) has no solution. Using (3.1) and (3.2) we derive that for each fixed index $p \in I$ the following system in the variable $x^{\prime}=(x, r) \in \mathbb{R}^{n} \times \mathbb{R}$ has no solution:

(III)

$$
\left\{\begin{array}{l}
\langle 0, x\rangle-1 r<0, \\
f_{p}(x)-b_{p} r<0, \\
f_{i}(x)-b_{i} r \leqq 0, \quad i \neq p, \\
g_{j}(x)-c_{j} r \leqq 0, \quad j \in J, \\
h_{s}(x)-a_{s} r \leqq 0, \quad s \in L, \\
\tilde{h}_{s}(x)+a_{s} r \leqq 0, \quad s \in L, \\
q(x)+0 r \leqq 0
\end{array}\right.
$$


By the closedness assumption and Lemma 3.1, we have

$$
\begin{aligned}
0 \in & \operatorname{co}\left\{B_{p} \times\left\{-b_{p}\right\},\{0\} \times\{-1\}\right\} \\
& +\operatorname{cone}\left\{\bigcup_{i \neq p} B_{i}^{\prime}, \bigcup_{j \in J} C_{j}, \bigcup_{s \in L} A_{s}^{\prime},-\bigcup_{s \in L} A_{s}^{\prime}\right\}+\left[S^{-} \times\{0\}\right] .
\end{aligned}
$$

Thus there exist $\lambda_{p}^{\prime} \geqq 0, r_{p}^{\prime} \geqq 0, \lambda_{i}^{(p)} \geqq 0,(i \neq p), \mu_{j}^{(p)} \geqq 0,(j \in J), \delta_{s}^{(p)} \geqq 0$, $\bar{\delta}_{s}^{(p)} \geqq 0,(s \in L)$ such that

$$
\begin{gathered}
r_{p}^{\prime}+\lambda_{p}^{\prime}=1 \\
0 \in r_{p}^{\prime} 0+\lambda_{p}^{\prime} B_{p}+\sum_{i \neq p} \lambda_{i}^{(p)} B_{i}+\sum_{i \in J} \mu_{j}^{(p)} C_{j} \\
+\sum_{s \in L} \delta_{s}^{(p)} A_{s}-\sum_{s \in L} \bar{\delta}_{s}^{(p)} A_{s}+S^{-}
\end{gathered}
$$

and

$$
\begin{aligned}
0= & -1 \cdot r_{p}^{\prime}-\lambda_{p}^{\prime} b_{p}-\sum_{i \neq p} \lambda_{i}^{(p)} b_{i}-\sum_{j \in J} \mu_{j}^{(p)} c_{j} \\
& -\sum_{s \in L} \delta_{s}^{(p)} a_{s}+\sum_{s \in L} \bar{\delta}_{s}^{(p)} a_{s}+0 .
\end{aligned}
$$

Summing up (3.11) over $p \in I$ and setting

$$
\begin{aligned}
\lambda_{1} & =\lambda_{1}^{\prime}+\lambda_{1}^{(2)}+\lambda_{1}^{(3)}+\cdots+\lambda_{1}^{(m)}, \\
\lambda_{2} & =\lambda_{2}^{(1)}+\lambda_{2}^{\prime}+\lambda_{2}^{(3)}+\cdots+\lambda_{2}^{(m)}, \\
& \cdots \cdots \cdots \cdots \cdots \cdots+\lambda_{m}^{\prime}, \\
\lambda_{m} & =\lambda_{m}^{(1)}+\lambda_{m}^{(2)}+\lambda_{m}^{(3)}+\cdots+\lambda_{m}^{\prime} \\
\mu_{j} & =\mu_{j}^{(1)}+\mu_{j}^{(2)}+\cdots+\mu_{j}^{(m)}, \quad j \in J, \\
\delta_{s} & =\delta_{s}^{(1)}+\delta_{s}^{(2)}+\cdots+\delta_{s}^{(l)}, \quad s \in L, \\
\bar{\delta}_{s} & =\bar{\delta}_{s}^{(1)}+\bar{\delta}_{s}^{(2)}+\cdots+\bar{\delta}_{s}^{(l)}, \quad s \in L,
\end{aligned}
$$

we obtain that

$$
0 \in \sum_{i \in I} \lambda_{i} B_{i}+\sum_{j \in J} \mu_{j} C_{j}+\sum_{s \in L} \delta_{s} A_{s}-\sum_{s \in L} \bar{\delta}_{s} A_{s}+S^{-}
$$

Summing up (3.12) $)_{p}$ over $p \in I$ and setting

$$
r^{\prime}=r_{1}^{\prime}+r_{2}^{\prime}+\cdots+r_{m}^{\prime}
$$


we obtain

$$
0=-1 \cdot r^{\prime}-\sum_{i \in I} \lambda_{i} b_{i}-\sum_{j \in J} \mu_{j} c_{j}-\sum_{s \in L} \delta_{s} a_{s}+\sum_{s \in L} \bar{\delta}_{s} a_{s}+0
$$

There are two cases:

(i) $\forall p \in I, r_{p}^{\prime}=0$ (hence $r^{\prime}=0$ by (3.14));

(ii) $\exists p \in I$ such that $r_{p}^{\prime}>0$ (hence $r^{\prime}>0$ by (3.14)).

In case (i): $\forall p \in I, \lambda_{p}^{\prime}=1\left(\operatorname{see}(3.10)_{p}\right)$. Therefore $\lambda_{p}>0(\forall p \in I)$ and system (I) has a solution.

In case (ii): system (II) has a solution (see (3.13), (3.15)).

(2) Suppose that $A_{s}$ is a singleton for any $s \in L$. Assume to the contrary that (a) and (b) hold simultaneously and $\lambda_{i}, \mu_{j}, \delta_{s}, \bar{\delta}_{s}$ satisfy system (I). Since $\left(\lambda_{i}\right)_{i \in I}>0$, we see from (3.3) that there exists $x \in S$ such that

$$
\begin{aligned}
\sum_{i \in I} \lambda_{i} f_{i}(x) & <\sum_{i \in I} \lambda_{i} b_{i}, & \sum_{s \in L} \delta_{s} h_{s}(x) & =\sum_{s \in L} \delta_{s} a_{s}, \\
\sum_{j \in J} \mu_{j} g_{j}(x) & \leqq \sum_{j \in J} \mu_{j} c_{j}, & -\sum_{s \in L} \bar{\delta}_{s} h_{s}(x) & =-\sum_{s \in L} \bar{\delta}_{s} a_{s}
\end{aligned}
$$

and hence we have

$$
\begin{aligned}
\zeta(x) & :=\sum_{i \in I} \lambda_{i} f_{i}(x)+\sum_{j \in J} \mu_{j} g_{j}(x)+\sum_{s \in L} \delta_{s} h_{s}(x)-\sum_{s \in L} \bar{\delta}_{s} h_{s}(x) \\
& <\sum_{i \in I} \lambda_{i} b_{i}+\sum_{j \in J} \mu_{j} c_{j}+\sum_{s \in L} \delta_{s} a_{s}-\sum_{s \in L} \bar{\delta}_{s} a_{s}=0 \quad \text { (by (3.6)). }
\end{aligned}
$$

On the other hand, since $A_{s}$ is a singleton, it follows from (3.5) that there exists $\xi \in S^{-}$such that

$$
\langle-\xi, x\rangle \leqq \zeta(x)
$$

Since $x \in S$ and $\xi \in S^{-}$, then $\langle-\xi, x\rangle \geqq 0$. Hence (3.17) implies that $\zeta(x) \geqq 0$, a contradiction to (3.16).

Assume now that $x$ satisfies (3.3), and $\lambda_{i}, \mu_{j}, \delta_{s}, \bar{\delta}_{s}$ satisfy (II). Then we have

$$
\begin{aligned}
0 & >\sum_{i \in I} \lambda_{i} b_{i}+\sum_{j \in J} \mu_{j} c_{j}+\sum_{s \in L} \delta_{s} a_{s}-\sum_{s \in L} \bar{\delta}_{s} a_{s} \quad \text { (by (3.9)) } \\
& \geqq \zeta(x) \quad(\text { by }(3.3) \text { and }(3.7)) \\
& \geqq\langle-\xi, x\rangle \quad(\text { by }(3.17)) \\
& \geqq 0 .
\end{aligned}
$$

This is a contradiction. 
REMARK 3.2. The closedness assumption $(\mathrm{H})$ is not used for proving that (a) and (b) do not hold simultaneously.

REMARK 3.3. If $A_{s}$ is a singleton for any $s \in L$, then (b) in Theorem 3.1 can be replaced by $(b)^{\prime}$, where

(b)

$$
\left\{\begin{array}{l}
\left(\lambda_{i}\right)_{i \in I}>0, \quad\left(\mu_{j}\right)_{j \in J} \geqq 0, \quad\left(\tilde{\delta}_{s}\right)_{s \in L} \in \mathbb{R}^{l}, \\
0 \in \sum_{i \in I} \lambda_{i} B_{i}+\sum_{j \in J} \mu_{j} C_{j}+\sum_{s \in L} \tilde{\delta}_{s} A_{s}+S^{-}, \\
0=\sum_{i \in I} \lambda_{k} b_{i}+\sum_{j \in J} \mu_{j} c_{j}+\sum_{s \in L} \tilde{\delta}_{s} a_{s}
\end{array}\right.
$$

or

$$
\left\{\begin{array}{l}
\left(\lambda_{i}\right)_{i \in I} \geqq 0, \quad\left(\mu_{j}\right)_{j \in J} \geqq 0, \quad\left(\tilde{\delta}_{s}\right)_{s \in L} \in \mathbb{R}^{l}, \\
0 \in \sum_{i \in I} \lambda_{i} B_{i}+\sum_{j \in J} \mu_{j} C_{j}+\sum_{s \in L} \tilde{\delta}_{s} A_{s}+S^{-}, \\
0>\sum_{i \in I} \lambda_{i} b_{i}+\sum_{j \in J} \mu_{j} c_{j}+\sum_{s \in L} \tilde{\delta}_{s} a_{s}
\end{array}\right.
$$

has a solution.

This can be obtained by setting $\tilde{\delta}_{s}=\delta_{s}-\tilde{\delta}_{s}\left(\tilde{\delta}_{s}\right.$ can be nonnegative or nonpositive).

COROLLARY 3.1. In addition to the closedness assumption $(\mathrm{H})$ of Theorem 3.1, we assume that the system

$$
f(x) \leqq b, \quad g(x) \leqq c, \quad h(x)=a \quad x \in S
$$

has at least a solution and for any $s \in L, A_{s}$ is a singleton.

Then either

(a) System (3.3) has a solution or

(b) System (I) has a solution,

but never both.

PROOF. It suffices to show that the consistency of (3.22) implies the inconsistency of (II). We omit the proof of this fact since it can be established by an argument similar to that used in the proof of the second part of Theorem 3.1 (see (3.18)-(3.21)).

COROLlARY 3.2. Let $S^{-}$be a polyhedral cone. Let each of the sets $B_{i}, C_{j}$ and $A_{s}$ be a singleton: $B_{i}=\left\{u_{i}\right\}, C_{j}=\left\{v_{j}\right\}$ and $A_{s}=\left\{w_{s}\right\}$. Denote by $\langle u, x\rangle$ the vector with components $\left\langle u_{i}, x\right\rangle(i \in I)$ and similarly for $\langle v, x\rangle$ and $\langle w, x\rangle$. 
(A) Then either

(a) the system

$$
\langle u, x\rangle \leq b, \quad\langle v, x\rangle \leqq c, \quad\langle w, x\rangle=a, \quad x \in S
$$

has a solution or

(b) the system

$(\mathrm{I})^{\prime}$

or

$$
\left\{\begin{array}{l}
\left(\lambda_{i}\right)_{i \in I}>0, \quad\left(\mu_{j}\right)_{j \in J} \geqq 0, \quad\left(\delta_{s}\right)_{s \in L} \in \mathbb{R}^{l}, \\
0 \in \sum_{i \in I} \lambda_{i} u_{i}+\sum_{j \in J} \mu_{j} v_{j}+\sum_{s \in L} \delta_{s} w_{s}+S^{-} \\
0=\sum_{i \in I} \lambda_{i} b_{i}+\sum_{j \in J} \mu_{j} c_{j}+\sum_{s \in L} \delta_{s} a_{s}
\end{array}\right.
$$

has a solution,

$$
\left\{\begin{array}{l}
\left(\lambda_{i}\right)_{i \in I} \geqq 0, \quad\left(\mu_{j}\right)_{j \in J} \geqq 0, \quad\left(\delta_{s}\right)_{s \in L} \in \mathbb{R}^{\prime}, \\
0 \in \sum_{i \in I} \lambda_{i} u_{i}+\sum_{j \in J} \mu_{j} v_{j}+\sum_{s \in L} \delta_{s} w_{s}+S^{-}, \\
0>\sum_{i \in I} \lambda_{i} b_{i}+\sum_{j \in J} \mu_{j} c_{j}+\sum_{s \in L} \delta_{s} a_{s}
\end{array}\right.
$$

but never both.

(B) If we additionally assume that the system

$$
\langle u, x\rangle \leqq b, \quad\langle v, x\rangle \leqq c, \quad\langle w, x\rangle=a, \quad x \in S
$$

has at least a solution, then either

(a) System (3.23) has a solution or

(b) System (I)' has a solution,

but never both.

PROOF. This is a direct consequence of Theorem 3.1, Corollary 3.1 and Remark 3.1.

REMARK 3.4. Let us compare our Corollary 3.2 with [23, Theorem 2.1] under the same assumption that $S=\mathbb{R}^{n}$ (which implies that $S^{-}=\{0\}$ ). In this special case, part (A) of Corollary 3.2 is quite different from Theorem 2.1 of $\mathrm{Xu}$ [23] since in our case the equalities exist. Also, unlike [23] no assumption is imposed on our corollary. Our conclusion is quite different from that of $\mathrm{Xu}$ [23] and does not contradict his counterexample 1.1 .

COROLlary 3.3. Let each of the sets $A_{s}$ be a singleton: $A_{s}=\left\{w_{s}\right\}$. Consider the' following statements: 
(a) System (3.3) has a solution.

(b) For any $u_{i} \in B_{i}(i \in I)$ and $v_{j} \in C_{j}(j \in J)$, system (3.23) has a solution.

Then $(\mathrm{a}) \Rightarrow(\mathrm{b})$; and the converse implication holds if the closedness assumption $(\mathrm{H})$ is satisfied.

PROOF. (a) $\Rightarrow$ (b) This implication is clear from the definition of the functions $f$ and $g$.

(b) $\Rightarrow$ (a) Assume to the contrary that system (3.3) has no solution. Then by Theorem 3.1 either system (I) or system (II) has a solution. If system (I) has a solution then there exist $u_{i} \in B_{i}$ and $v_{j} \in C_{j}$ such that system (I)' has a solution. By Theorem 3.1 and Remark 3.2, system (3.23) has no solution, a contradiction to statement (b). Similarly, the consistency of system (II) implies the consistency of system (II)' where $u_{i} \in B_{i}$ and $v_{j} \in C_{j}$ are suitable points. By Theorem 3.1 and Remark 3.2, system (3.23) has no solution, a contradiction to statement (b).

Now we will consider an application of Corollary 3.1 to a concave vector optimisation problem. Let $f=\left(f_{1}, f_{2}, \ldots, f_{m}\right)$ and $g=\left(g_{1}, g_{2}, \ldots, g_{k}\right)$ be vector-valued maps with components being concave on $\mathbb{R}^{n}$. This means that for all $\bar{x}$ and $x_{0} \in \mathbb{R}^{n}$

$$
\begin{aligned}
& f_{i}(\bar{x})-f_{i}\left(x_{0}\right) \leqq f_{i}^{0}\left(x_{0} ; \bar{x}-x_{0}\right) \quad(i=1,2, \ldots, m), \\
& g_{j}(\bar{x})-g_{j}\left(x_{0}\right) \leqq g_{j}^{0}\left(x_{0} ; \bar{x}-x_{0}\right) \quad(j=1,2, \ldots, k) .
\end{aligned}
$$

Let $S$ and $M$ be closed convex cones in $\mathbb{R}^{n}$ and $\mathbb{R}^{l}$, respectively. Let $A$ be an $l \times n$-matrix, and let $c=\left(c_{1}, c_{2}, \ldots, c_{k}\right)$ and $d=\left(d_{1}, d_{2}, \ldots, d_{l}\right)$ be given vectors.

Consider the following concave vector optimisation problem $(V O P)_{C}$ with concave constraints and abstract linear constraints:

$(V O P)_{C}$

$$
\begin{aligned}
\text { Minimise } & f(x):=\left(f_{1}(x), f_{2}(x), \ldots, f_{m}(x)\right) \\
\text { subject to } & g(x) \leqq c \\
& A x+d \in M, \\
& x \in S .
\end{aligned}
$$

A point $x$ satisfying (3.26)-(3.28) is called a feasible solution of $(V O P)_{C}$. We are interested in finding an efficient solution $x_{0}$ of $(V O P)_{c}$, that is, a feasible solution $x_{0}$ such that there is no other feasible solution $x$ of $(V O P)_{C}$ with $f(x) \leq f\left(x_{0}\right)$. Obviously, if $x_{0} \in S$ is an efficient solution for $(V O P)_{c}$, then the system

$$
f^{0}\left(x_{0} ; x\right) \leq 0, \quad g^{0}\left(x_{0} ; x\right) \leqq c^{\prime}, \quad A x+d^{\prime} \in M, \quad x \in S
$$

is inconsistent where $c^{\prime}=c-g\left(x_{0}\right)$ and $d^{\prime}=A x_{0}+d$. Indeed, if this system has a solution $x$ then in view of (3.24) and (3.25) we see that $\bar{x}:=x+x_{0}$ is a feasible 
solution of $(V O P)_{C}$ such that $f(\bar{x}) \leq f\left(x_{0}\right)$, a contradiction to the efficiency property of $x_{0}$.

Let us set $x^{\prime}=(x, y) \in \mathbb{R}^{n} \times \mathbb{R}^{l}$,

$$
\begin{array}{rlrl}
F_{i}\left(x^{\prime}\right): & =f_{i}^{0}\left(x_{0} ; x\right)=\max _{\xi^{\prime} \in B_{i}^{\prime}}\left\langle\xi^{\prime}, x^{\prime}\right\rangle, & H_{s}\left(x^{\prime}\right) & =\left\langle a_{s}^{\prime}, x^{\prime}\right\rangle, \\
G_{j}\left(x^{\prime}\right):=g_{j}^{0}\left(x_{0} ; x\right)=\max _{\xi^{\prime} \in C_{j}}\left\langle\xi^{\prime}, x^{\prime}\right\rangle, & S^{\prime}=S \times M,
\end{array}
$$

where $B_{i}^{\prime}=\partial f_{i}\left(x_{0}\right) \times\{0\}, C_{j}^{\prime}=\partial g_{j}\left(x_{0}\right) \times\{0\}, a_{s}^{\prime}=\left(a_{s},-p_{s}\right), a_{s}$ is the $s$-th row of the matrix $A$ and $p_{s}=(0, \ldots, 0,1,0, \ldots, 0) \in \mathbb{R}^{l}$ (1 being the $s$-th component of $p_{s}$ ).

Then the following system must be inconsistent:

$$
F\left(x^{\prime}\right) \leq 0, \quad G\left(x^{\prime}\right) \leqq c^{\prime}, \quad H\left(x^{\prime}\right)=-d^{\prime}, \quad x^{\prime} \in S^{\prime} .
$$

Observe that $x^{\prime}=\left(0, d^{\prime}\right) \in \mathbb{R}^{n} \times \mathbb{R}^{l}$ is a solution of the last system with the sign $\leq$ of its first inequality being replaced by $\leqq$. So by Corollary 3.1 , we find $\left(\lambda_{i}\right)_{i \in I}>0$, $\left(\mu_{j}\right)_{j \in J} \geqq 0$ and $r_{s} \in \mathbb{R}(s \in L)$ such that

$$
0 \in \sum_{i \in I} \lambda_{i} B_{i}^{\prime}+\sum_{j \in J} \mu_{j} C_{j}^{\prime}+\sum_{s \in L} r_{s} a_{s}^{\prime}+S^{\prime-}
$$

and

$$
0=\sum_{i \in I} \lambda_{i} 0+\sum_{j \in J} \mu_{j} c_{j}^{\prime}-\sum_{s \in L} r_{s} d_{s}^{\prime}
$$

if the following closedness assumption holds: for every $i \in I$, the set

$$
Q(i)=\text { cone }\left\{\bigcup_{i^{\prime} \neq i} B_{i^{\prime}}^{\prime} \times\{0\}, \bigcup_{j \in J} C_{j}^{\prime} \times\left\{-c_{j}\right\}\right\}+\mathrm{sp}\left[\bigcup_{s \in L}\left\{a_{s}^{\prime}\right\} \times\left\{d_{s}^{\prime}\right\}\right]+\left[S^{\prime-} \times\{0\}\right]
$$

is closed.

From (3.29) and the definitions of $B_{i}^{\prime}, C_{j}^{\prime}$ and $a_{s}^{\prime}$, we can derive that

$$
0 \in \sum_{i \in l} \lambda_{i} \partial f_{i}\left(x_{0}\right)+\sum_{j \in J} \mu_{j} \partial g_{j}\left(x_{0}\right)+\sum_{s \in L} r_{s} a_{s}+S^{-}
$$

and

$$
0=\sum_{i \in I} \lambda_{i} 0+\sum_{j \in J} \mu_{j} 0-\sum_{s \in L} r_{s} p_{s}+M^{-} .
$$

Setting $r=\left(r_{1}, r_{2}, \ldots, r_{l}\right)$, we see that $\sum_{k \in L} r_{k} p_{k}=r$. Thus (3.32) means that $r \in M^{-}$. From (3.30), we have

$$
\sum_{j \in J} \mu_{j}\left(c_{j}-g_{j}\left(x_{0}\right)\right)-\sum_{s \in L}\left[r_{s}\left\langle a_{s}, x_{0}\right\rangle+r_{s} d_{s}\right]=0
$$


(from which we can derive the complementarity condition).

From the above discussion, we can obtain the following necessary optimality condition for $(V O P)_{C}$.

THEOREM 3.2. Assume that for every $i \in I$ the set $Q(i)$ is closed. If $x_{0}$ is an efficient solution of the concave vector optimisation problem $(V O P)_{C}$, then there are $\left(\lambda_{i}\right)_{i \in I}>0,\left(\mu_{j}\right)_{j \in J} \geqq 0$ and $\left(r_{s}\right)_{s \in L} \in M^{-}$satisfying (3.31) and (3.33).

COROLLARY 3.4. In addition to the above assumption that $Q(i)$ is closed for each $i \in I$, assume that $g \equiv 0, c=0$ and $f$ is of class $C^{1}$. If $x_{0}$ is an efficient solution for $(V O P)_{C}$, then there are $\left(\lambda_{i}\right)_{i \in I}>0,\left(r_{s}\right)_{s \in L} \in M^{-}$such that

$$
0 \in \sum_{i \in I} \lambda_{i} f_{i x_{0}}^{\prime}+\sum_{s \in L} r_{s} a_{s}+S^{-} \text {and } 0=\sum_{s \in L} r_{s}\left(\left\langle a_{s}, x_{0}\right\rangle+d_{s}\right),
$$

where $f_{i x_{0}}^{\prime}$ is the Fréchet derivative of $f_{i}$ at $x_{0}$.

REMARK 3.5. In the case where $M=\mathbb{R}_{-}^{l}$ (the nonpositive orthant of $\mathbb{R}^{l}$ ) and $S=\mathbb{R}^{n}$, Corollary 3.4 is exactly Theorem 3.1 of [23] (the closedness assumption is automatically satisfied since the sum of polyhedral cones is closed).

\section{Efficient solutions of nonsmooth problems of vector optimisation}

In this section we will use the notion of a Kuhn-Tucker point for the vector optimisation problem (VOP) which coincides with the usual notion of a Kuhn-Tucker point for the case of scalar optimisation. For smooth problems with inequality constraints only, Martin [13] (see also [14,15]) introduced a class of KT-invex problems and proved that every Kuhn-Tucker point is a global minimiser if and only if this problem is KT-invex. The main result of this section is Theorem 4.1 which shows that the above result of Martin can be extended to the case of nonsmooth vector optimisation problems with mixed constraints, that is, the case when not only inequality constraints (1.1) but also equality constraints (1.2) and a "geometric" constraint (1.3) are considered. The class of KT-pseudoinvex-infine problems (Definition 4.1) and the class of GKT-pseudoinvex-infine problems (Definition 4.1') will be used as substitutes for the class of KT-invex problems of Martin. We will see that they are suitable for our goal. As a consequence of Theorem 4.1 we will obtain a generalisation of the above result of Martin to problems with mixed constraints (see Remark 4.3). This section will also discuss relationships between several classes of invex-infine problems (see Theorems 4.2 and 4.3).

Let $f:=\left(f_{1}, f_{2}, \ldots, f_{m}\right): \mathbb{R}^{n} \rightarrow \mathbb{R}^{m}, g:=\left(g_{1}, g_{2}, \ldots, g_{k}\right): \mathbb{R}^{n} \rightarrow \mathbb{R}^{k}$ and $h:=$ $\left(h_{1}, h_{2}, \ldots, h_{l}\right): \mathbb{R}^{n} \rightarrow \mathbb{R}^{l}$ be locally Lipschitz functions, and let $S$ be a nonempty closed subset of $\mathbb{R}^{n}$. Let $I=\{1,2, \ldots, m\}, J=\{1,2, \ldots, k\}$ and $L=\{1,2, \ldots, l\}$. 
Consider the vector optimisation problem (VOP) formulated in the introduction:

Minimise $f(x)$ subject to $x \in S_{1}$,

where $S_{1}$ denotes the set of all points $x$ satisfying (1.1)-(1.3). We will be interested in efficient solutions of (VOP). Recall that $x_{0} \in S_{1}$ is an efficient solution of (VOP) if for any $x \in S_{1}, f(x) \leq f\left(x_{0}\right)$, that is, there does not exist $x \in S_{1}$ such that $f_{i}(x) \leqq f_{i}\left(x_{0}\right)$ for all $i$ and at least one of these inequalities is strict.

Let $J_{0}=\left\{j \in J: g_{j}\left(x_{0}\right)=0\right\}$. A point $x_{0} \in S_{1}$ is said to be a Kuhn-Tucker point of $(V O P)$ if there are vectors $\left(\lambda_{i}\right)_{i \in I}>0,\left(\mu_{j}\right)_{j \in J_{0}} \geqq 0$ and $\left(r_{s}\right)_{s \in L} \in \mathbb{R}^{l}$ such that

$$
0 \in \sum_{i \in I} \lambda_{i} \partial f_{i}\left(x_{0}\right)+\sum_{j \in J_{0}} \mu_{j} \partial g_{j}\left(x_{0}\right)+\sum_{s \in L} r_{s} \partial h_{s}\left(x_{0}\right)+N_{S}\left(x_{0}\right)
$$

This becomes the usual notion of a Kuhn-Tucker point if $m=1$.

Definition 4.1. Problem (VOP) is KT-pseudoinvex-infine at $x_{0} \in S_{1}$ if for any $x \in S_{1}$ with $f(x) \leq f\left(x_{0}\right)$ there is $\eta \in T_{S}\left(x_{0}\right)$ such that

$$
\begin{aligned}
& 0 \geq f^{0}\left(x_{0} ; \eta\right), \\
& 0 \geqq g_{J_{0}}^{0}\left(x_{0} ; \eta\right), \\
& 0=h^{0}\left(x_{0} ; \eta\right) .
\end{aligned}
$$

REMARK 4.1. Let us consider problem (VOP) when the function $h$ is absent. In this case, it is natural to use the terminology "KT-pseudoinvex" instead of "KTpseudoinvex-infine". We now provide an example showing that (VOP) is KTpseudoinvex in the above sense but it is not KT-pseudoinvex in the weaker sense of [19]. Recall that the authors of [19] say that the problem (VOP) of minimising $f$ subject to $g(x) \leqq 0, x \in S$ is KT-pseudoinvex at $x_{0} \in S_{1}:=\{x \in S: g(x) \leqq 0\}$ if for any $x \in S_{1}$ with $f(x)<f\left(x_{0}\right)$ there exists $\eta \in T_{S}\left(x_{0}\right)$ such that

$$
0>f^{0}\left(x_{0} ; \eta\right), \quad 0 \geqq g_{J_{0}}^{0}\left(x_{0} ; \eta\right) .
$$

Consider the following vector optimisation problem:

$$
\text { Minimise } f(x) \text { subject to } x \in S_{1}:=\{x \in S: g(x) \leqq 0\},
$$

where $f(x)=\left(-x^{2}, x^{2}-x\right), g(x)=\left(x^{2}-1 / 4,-x\right)$ and $S=\mathbb{R}$. Then $S_{1}=[0,1 / 2]$. For $x_{0}=0$, the active constraint function is $g_{2}(x)=-x$ and we have $f_{x_{0}}^{\prime}=(0,-1)$ and $g_{2 x_{0}}^{\prime}=-1$. We can check that $f(x)<f\left(x_{0}\right)$ for any $x \in S_{1} \backslash\{0\}$. For all such $x$, let $\eta=1$. Then we have $f_{x_{0}}^{\prime} \eta=(0,-\eta) \leq 0$ and $g_{2 x_{0}}^{\prime} \eta=-\eta<0$. Thus (VOP) is KT-pseudoinvex, but (VOP) is not KT-pseudoinvex in the sense of [19] since $f_{1_{x_{0}}}^{\prime}=0$ and hence we cannot find $\eta$ such that $f_{x_{0}}^{\prime} \eta<0$. 
DEFINITION 4.1'. Problem (VOP) is GKT-pseudoinvex-infine at $x_{0} \in S_{1}$ if for any $x \in S_{1}$ with $f(x) \leq f\left(x_{0}\right)$ and

$$
u_{i} \in \partial f_{i}\left(x_{0}\right)(i \in I), \quad v_{j} \in \partial g_{j}\left(x_{0}\right)\left(j \in J_{0}\right), \quad w_{s} \in \partial h_{s}\left(x_{0}\right)(s \in L),
$$

there is $\eta \in T_{S}\left(x_{0}\right)$ such that

$$
\begin{aligned}
& 0 \geqq\langle u, \eta\rangle, \\
& 0 \geqq\langle v, \eta\rangle, \\
& 0=\langle w, \eta\rangle .
\end{aligned}
$$

(Recall that $\langle u, \eta\rangle$ is the vector with components $\left\langle u_{i}, \eta\right\rangle(i \in I)$ and similarly for $\langle v, \eta\rangle$ and $\langle w, \eta\rangle$.)

Let us introduce the following closedness assumptions.

$\left(\mathrm{H}_{1}\right)$ For any $i \in I$ the set

$$
\text { cone }\left\{\bigcup_{i^{\prime} \neq i} \partial f_{i^{\prime}}\left(x_{0}\right), \bigcup_{j \in J_{0}} \partial g_{j}\left(x_{0}\right)\right\}+\operatorname{sp} \bigcup_{s \in L} \partial h_{s}\left(x_{0}\right)+N_{S}\left(x_{0}\right)
$$

is closed.

$\left(\mathrm{H}_{1}\right)^{\prime}$ For any $i \in I$ and

$$
u_{i^{\prime}} \in \partial f_{i^{\prime}}\left(x_{0}\right)\left(i^{\prime} \neq i\right), \quad v_{j} \in \partial g_{j}\left(x_{0}\right)\left(j \in J_{0}\right), \quad w_{s} \in \partial h_{s}\left(x_{0}\right)(s \in L),
$$

the set cone $\left\{\bigcup_{i^{\prime} \neq i} u_{i^{\prime}}, \bigcup_{j \in J_{0}} v_{j}\right\}+\operatorname{sp} \bigcup_{s \in L} w_{s}+N_{s}\left(x_{0}\right)$ is closed.

THEOREM 4.1. Consider the following statements:

(a) Problem (VOP) is KT-pseudoinvex-infine at $x_{0} \in S_{1}$;

(b) Problem (VOP) is GKT-pseudoinvex-infine at $x_{0} \in S_{1}$;

(c) If $x_{0} \in S_{1}$ is a Kuhn-Tucker point then $x_{0}$ is an efficient solution of problem (VOP).

Then

(1) (a) $\Rightarrow$ (b) if $h$ is of class $C^{1}$; and (b) $\Rightarrow$ (a) if $h$ is of class $C^{1}$ and $\left(\mathrm{H}_{1}\right)$ holds.

(2) (b) $\Rightarrow$ (c); and (c) $\Rightarrow$ (b) if $\left(\mathrm{H}_{1}\right)^{\prime}$ holds.

(3) (a) $\Rightarrow$ (c) if $h$ is of class $C^{1}$; and (c) $\Rightarrow$ (a) if $h$ is of class $C^{1}$ and $\left(\mathrm{H}_{1}\right)$ holds.

Proof. (a) $\Rightarrow$ (b) (if $h$ is of class $C^{1}$ ): Obviously. (Observe that $\partial h_{s}\left(x_{0}\right)$ equals the Fréchet derivative $h_{s x_{0}}^{\prime}$ of $h_{s}$ at $x_{0}$ since $h_{s}$ is of class $C^{\mathbf{l}}$.)

(b) $\Rightarrow$ (a) (if $h$ is of class $C^{1}$ and assumption $\left(\mathrm{H}_{1}\right)$ holds): This is a direct consequence of Definitions $4.1,4.1^{\prime}$ and Corollary 3.3 where $S$ is replaced by $N_{S}\left(x_{0}\right), J$ is replaced by $J_{0}$ and functions $f_{i}, g_{j}$ and $h_{s}$ are replaced by

$$
\overline{f_{i}}(\cdot)=f_{i}^{0}\left(x_{0} ; \cdot\right), \quad \bar{g}_{j}(\cdot)=g_{j}^{0}\left(x_{0} ; \cdot\right), \quad \bar{h}_{s}(\cdot)=h_{s}^{0}\left(x_{0} ; \cdot\right)
$$


(b) $\Rightarrow$ (c): Let $\lambda_{i}>0(i \in I), \mu_{j} \geqq 0,\left(j \in J_{0}\right), r_{s} \in \mathbb{R},(s \in L), u_{i} \in \partial f_{i}\left(x_{0}\right)$, $v_{j} \in \partial g_{j}\left(x_{0}\right), w_{s} \in \partial h_{s}\left(x_{0}\right)$ and $y \in N_{S}\left(x_{0}\right)$ be such that

$$
\zeta:=\sum_{i \in I} \lambda_{i} u_{i}+\sum_{j \in J_{0}} \mu_{j} v_{j}+\sum_{s \in L} r_{s} w_{s}=-y,
$$

that is, let $x_{0}$ be a Kuhn-Tucker point of (VOP). Suppose to the contrary that $x_{0} \in S_{1}$ is not efficient for $(V O P)$. Then $f(x) \leq f\left(x_{0}\right)$ for some $x \in S_{1}$. From (4.2)'-(4.4)', we have $\langle\zeta, \eta\rangle<0$ for suitable $\eta \in T_{S}\left(x_{0}\right)$ while (4.8) yields $\langle\zeta, \eta\rangle=(-y, \eta\rangle \geqq 0$. Thus we obtain a contradiction and the efficiency of $x_{0}$ is proved.

(c) $\Rightarrow$ (b) (if $\left(\mathrm{H}_{1}\right)^{\prime}$ holds): If $x_{0} \in S_{1}$ is a Kuhn-Tucker point of (VOP), then by statement (c), there is no $x \in S_{1}$ such that $f(x) \leq f\left(x_{0}\right)$. This obviously means that $(V O P)$ is GKT-pseudoinvex-infine at $x_{0}$.

Assume now that $x_{0} \in S_{1}$ is not a Kuhn-Tucker point of (VOP). Then, for any $u_{i}, v_{j}$ and $w_{s}$ satisfying (4.5), the following system has no solution:

$$
\begin{gathered}
\left(\lambda_{i}\right)_{i \in I}>0, \quad\left(\mu_{j}\right)_{j \in J_{0}} \geqq 0, \quad r_{s} \in \mathbb{R} \quad(s \in L), \\
0 \in \sum_{i \in I} \lambda_{i} u_{i}+\sum_{j \in J_{0}} \mu_{j} v_{j}+\sum_{s \in L} r_{s} w_{s}+N_{s}\left(x_{0}\right) .
\end{gathered}
$$

Observe that the system

$$
\langle u, x\rangle \leqq 0, \quad\langle v, x\rangle \leqq 0, \quad\langle w, x\rangle=0, \quad x \in T_{S}\left(x_{0}\right)
$$

has a solution $x=0$. By Corollary 3.1 the system

$$
\langle u, x\rangle \leq 0, \quad\langle v, x\rangle \leqq 0, \quad\langle w, x\rangle=0, \quad x \in T_{S}\left(x_{0}\right)
$$

has a solution denoted by $\eta$. Thus, for any $x \in S_{1}$ with $f(x) \leq f\left(x_{0}\right)$, the point $\eta$ satisfies all the requirements of Definition 4.1'.

(a) $\Rightarrow$ (c) (if $h$ is of class $C^{1}$ ): This is obvious since (a) $\Rightarrow$ (b) and (b) $\Rightarrow$ (c).

(c) $\Rightarrow$ (a) (if $h$ is of class $C^{1}$ and if $\left(\mathrm{H}_{1}\right)$ holds): If $x_{0}$ is a Kuhn-Tucker point then the conclusion is obvious since by statement (c) there is no $x \in S_{1}$ with $f(x) \leq f\left(x_{0}\right)$. If $x_{0}$ is not a Kuhn-Tucker point then the system

$$
\begin{gathered}
\left(\lambda_{i}\right)_{i \in I}>0, \quad\left(\mu_{j}\right)_{j \in J_{0}} \geqq 0, \quad r_{s} \in \mathbb{R} \quad(s \in L), \\
0 \in \sum_{i \in I} \lambda_{i} \partial f_{i}\left(x_{0}\right)+\sum_{j \in J_{0}} \mu_{j} \partial g_{j}\left(x_{0}\right)+\sum_{s \in L} r_{s} h_{s x_{0}}^{\prime}+N_{S}\left(x_{0}\right)
\end{gathered}
$$

has no solution. Observe that the system

$$
\overline{f_{i}}(x) \leqq 0(i \in I), \quad \bar{g}_{j}(x) \leqq 0\left(j \in J_{0}\right), \quad \bar{h}_{s}(x)=0(s \in L), x \in T_{S}\left(x_{0}\right)
$$


has a solution $x=0$, where $\bar{f}_{i}, \bar{g}_{j}$ and $\bar{h}_{s}$ are defined by (4.7). By Corollary 3.1, with $\overline{f_{i}}, \bar{g}_{j}, h_{s}$ and $T_{S}\left(x_{0}\right)$ in place of $f_{i}, g_{j}, h_{s}$ and $S$ respectively, we infer that system

$$
\bar{f}(x) \leq 0, \quad \bar{g}(x) \leqq 0, \quad \bar{h}(x)=0, \quad x \in T_{S}\left(x_{0}\right)
$$

has a solution $x=\eta$. Thus for any $x \in S_{1}$ with $f(x) \leq f\left(x_{0}\right)$ the point $\eta$ satisfies all the requirements of Definition 4.1 .

THEOREM 4.1'. (1) Assume that $h$ is of class $C^{1}$ and the closedness assumption $\left(\mathrm{H}_{1}\right)$ holds. Then the statements (a), (b) and (c) of Theorem 4.1 are equivalent.

(2) If $N_{S}\left(x_{0}\right)$ is a polyhedral cone then the statements (b) and (c) of Theorem 4.1 are equivalent.

PROOF. This is a consequence of Theorem 4.1 .

Before going further let us introduce some notation which is close to Definitions 4.1 and 4.1' (see Propositions 4.1 and 4.2).

Let $\bar{S}_{1}=\left\{x \in S_{1}: f_{i}(x)<f_{i}\left(x_{0}\right)\right.$ for some $\left.i \in I\right\}$.

Definition 4.2. Problem (VOP) is KT-invex-infine at $x_{0} \in S_{1}$ if for any $x \in \bar{S}_{1}$ there exists $\eta \in T_{S}\left(x_{0}\right)$ such that

$$
\begin{aligned}
f(x)-f\left(x_{0}\right) & \geq f^{0}\left(x_{0} ; \eta\right), \\
0 & \geqq g_{J_{0}}^{0}\left(x_{0} ; \eta\right), \\
0 & =h^{0}\left(x_{0} ; \eta\right) .
\end{aligned}
$$

Definition 4.2'. Problem (VOP) is GKT-invex-infine at $x_{0} \in S_{1}$ if for any $x \in \bar{S}_{1}$ and $u_{i}, v_{j}, w_{s}$ satisfying (4.5) there exists $\eta \in T_{s}\left(x_{0}\right)$ such that

$$
\begin{aligned}
f(x)-f\left(x_{0}\right) & \geq\langle u, \eta\rangle, \\
0 & \geqq\langle v, \eta\rangle, \\
0 & =\langle w, \eta\rangle .
\end{aligned}
$$

Definition 4.3. Problem (VOP) is HC-invex-infine at $x_{0} \in S_{1}$ if for any $x \in \bar{S}_{1}$ there exists $\eta \in T_{S}\left(x_{0}\right)$ such that

$$
\begin{aligned}
f(x)-f\left(x_{0}\right) & \geq f^{0}\left(x_{0} ; \eta\right), \\
g_{J_{0}}(x)-g_{J_{0}}\left(x_{0}\right) & \geqq g_{J_{0}}^{0}\left(x_{0} ; \eta\right), \\
0 & =h^{0}\left(x_{0} ; \eta\right) .
\end{aligned}
$$


Definition 4.3'. Problem (VOP) is GHC-invex-infine at $x_{0} \in S_{1}$ if for any $x \in \bar{S}_{1}$ and $u_{i}, v_{j}, w_{s}$ satisfying (4.5) there exists $\eta \in T_{S}\left(x_{0}\right)$ such that

$$
\begin{aligned}
f(x)-f\left(x_{0}\right) & \geqq\langle u, \eta\rangle, \\
g_{J_{0}}(x)-g_{J_{0}}\left(x_{0}\right) & \geqq\langle v, \eta\rangle, \\
0 & =\langle w, \eta\rangle .
\end{aligned}
$$

REMARK 4.2. Let us note [21] that the appearance of equality constraints (1.2) in problem $(V O P)$ leads to the introduction of a subclass of invex functions, called the class of infine functions. Recall [21] that a locally Lipschitz function $\tilde{\zeta}: \mathbb{R}^{n} \rightarrow \mathbb{R}$ is called infine on $S$ at $x_{0} \in S$ if

$$
\forall x \in S \quad \forall \xi \in \partial \tilde{\zeta}\left(x_{0}\right) \quad \exists \eta \in T_{S}\left(x_{0}\right): \quad \tilde{\zeta}(x)-\tilde{\zeta}\left(x_{0}\right)=\langle\xi, \eta\rangle .
$$

Consider now the trivial cone $\tilde{M}=\{0\}$ of $\mathbb{R}$ and define the $\tilde{M}$-invexity of $\tilde{\zeta}$ on $S$ at $x_{0}$ in the sense of Craven [6] (see also [17]) by requiring that

$$
\forall x \in S \quad \exists \eta \in T_{S}\left(x_{0}\right): \quad \tilde{\zeta}(x)-\tilde{\zeta}\left(x_{0}\right)-\tilde{\zeta}^{0}\left(x_{0} ; \eta\right) \in \tilde{M}
$$

(that is, $\tilde{\zeta}(x)-\bar{\zeta}\left(x_{0}\right)=\tilde{\zeta}^{0}\left(x_{0} ; \eta\right)$ ).

It is natural to ask if the class of infine functions coincides with the class of $\{0\}$-invex functions of Craven. The answer is negative: the function $\tilde{\zeta}(x)=|x|$ is $\{0\}$-invex on $S=\mathbb{R}$ at $x_{0}=0$ but it is not infine in our sense. So a separate definition of infine functions is needed. The situation is similar to that of the non-coincidence of the class of convex functions and the class of linear functions.

When dealing with both inequality and equality constraints (1.1) and (1.2) it is natural to require that each component of $g$ is invex and each component of $h$ is infine, with the same $\eta \in T_{S}\left(x_{0}\right)$. This is nothing more than the notion of invex-infineness of a vector-valued function introduced in [21]. Recall [21] that the vector-valued function $\left(g_{J_{0}} ; h\right)$ is called invex-infine on $S$ at $x_{0} \in S$ if for any $x \in S, v_{j} \in \partial g_{j}\left(x_{0}\right)\left(j \in J_{0}\right)$ and $w_{s} \in \partial h_{s}\left(x_{0}\right)(s \in L)$ there exists $\eta \in T_{S}\left(x_{0}\right)$ such that $g_{J_{0}}(x)-g_{J_{0}}\left(x_{0}\right) \geqq\langle v, \eta\rangle$ and $h(x)-h\left(x_{0}\right)=\langle w, \eta\rangle$. If in Definition 4.3' $\bar{S}_{1}=S_{1}$ then conditions (4.13) ${ }^{\prime}$ and (4.14)' mean that $\left(g_{0} ; h\right)$ is invex-infine on $S_{1}$ at $x_{0} \in S_{1}$ in the above sense. Indeed, for $x \in S_{1}$ we have $h(x)-h\left(x_{0}\right)=0$ and hence a combination of this equality with (4.13)' and (4.14)' shows that all the requirements of the definitions of invex-infineness of $\left(g_{J_{0}} ; h\right)$ are fulfilled.

We begin our discussion of the relationship between Definitions 4.1-4.3, and Definitions $4.1^{\prime}-4.3^{\prime}$ with the following obvious result.

PROPOSITION 4.1.

(1) $H C$-invex-infine $\Rightarrow K T$-invex-infine $\Rightarrow K T$-pseudo-invex-infine. 
(2) $G H C$-invex-infine $\Rightarrow G K T$-invex-infine $\Rightarrow G K T$-pseudoinvex-infine.

The following proposition shows that if $m=1$ then KT-invex-infine $\Leftrightarrow \mathrm{KT}$ pseudoinvex-infine, and GKT-invex-infine $\Leftrightarrow$ GKT-pseudoinvex-infine.

PROPOSITION 4.2. Assume that $m=1$ (that is, $f$ is a real-valued function). Then

(1) The following statements are equivalent:

(a) Problem (VOP) is $K T$-pseudoinvex-infine at $x_{0} \in S_{1}$.

(b) Problem (VOP) is KT-invex-infine at $x_{0} \in S_{1}$.

(c) For any $x \in S_{1}$ there exists $\eta \in T_{S}\left(x_{0}\right)$ such that

$$
\begin{aligned}
f(x)-f\left(x_{0}\right) & \geqq f^{0}\left(x_{0} ; \eta\right), \\
0 & \geqq g_{J_{0}}^{0}\left(x_{0} ; \eta\right), \\
0 & =h^{0}\left(x_{0} ; \eta\right) .
\end{aligned}
$$

(2) The following statements are equivalent:

(a) ${ }^{\prime}$ Problem (VOP) is GKT-pseudoinvex-infine at $x_{0} \in S_{1}$.

(b)' Problem (VOP) is GKT-invex-infine at $x_{0} \in S_{1}$.

(c) For any $x \in S_{1}$ and $u_{i}, v_{j}$, ws satisfying (4.5) there exists $\eta \in T_{S}\left(x_{0}\right)$ such that

$$
\begin{aligned}
f(x)-f\left(x_{0}\right) & \geqq\langle u, \eta\rangle, \\
0 & \geqq\langle v, \eta\rangle, \\
0 & =\langle w, \eta\rangle .
\end{aligned}
$$

PROOF. Let us prove the first part of Proposition 4.1. The second part can be proved using a similar argument.

(a) $\Rightarrow$ (b): Since $m=1, \bar{S}_{1}=\left\{x \in S_{1}: f(x)<f\left(x_{0}\right)\right\}$. Let $x \in \bar{S}_{1}$, that is, $f(x)<f\left(x_{0}\right)$. By Definition 4.1 there exists $\eta \in T_{S}\left(x_{0}\right)$ satisfying (4.3), (4.4) and the inequality $f^{0}\left(x_{0} ; \eta\right)<0$. Let $\gamma>0$ be such that

$$
f(x)-f\left(x_{0}\right)>\gamma f^{0}\left(x_{0} ; \eta\right)=f^{0}\left(x_{0} ; \bar{\eta}\right),
$$

where $\bar{\eta}=\gamma \eta \in T_{S}\left(x_{0}\right)$. Thus (4.9)-(4.11) hold, with $\bar{\eta}$ in place of $\eta$.

(b) $\Rightarrow$ (a): Since $m=1, f(x) \leq f\left(x_{0}\right) \Leftrightarrow f(x)<f\left(x_{0}\right)$ (see Section 2). Thus if $x \in S_{1}$ and $f(x) \leq f\left(x_{0}\right)$ then $x \in \bar{S}_{1}$. To complete the proof it remains to note that conditions (4.9)-(4.11) imply conditions (4.2)-(4.4).

(c) $\Rightarrow$ (b): Let $x \in S_{1}$ with $f(x) \leq f\left(x_{0}\right)$. Then by (c) there exists $\eta \in T_{S}\left(x_{0}\right)$ satisfying (4.15)-(4.17). Since $f(x) \leq f\left(x_{0}\right) \Leftrightarrow f(x)-f\left(x_{0}\right)<0$ we derive from (4.15) that $f^{0}\left(x^{0} ; \eta\right)<0$. As in the proof of implication (a) $\Rightarrow$ (b) we can find $\gamma>0$ such that (4.9)-(4.11) are satisfied, with $\eta$ being replaced by $\bar{\eta}=\gamma \eta \in T_{S}\left(x_{0}\right)$. 
(b) $\Rightarrow$ (c): If $x \in \bar{S}_{1}$ then the fact of the existence of $\eta \in T_{S}\left(x_{0}\right)$ satisfying (4.15)-(4.17) is a direct consequence of Definition 4.2. If $x \notin \bar{S}_{1}$, that is, $x \in S_{1}$ and $f(x) \geqq f\left(x_{0}\right)$, then $\eta=0 \in T_{S}\left(x_{0}\right)$ satisfies (4.15)-(4.17).

COROLLARY 4.1. Let $S=\mathbb{R}^{n}$. Let $f$ be a real-valued function and $f, g$ and $h$ be of class $C^{1}$. Then the following statements are equivalent:

(a) For any $x \in S_{1}$ there is $\eta \in \mathbb{R}^{n}$ such that

$$
f(x)-f\left(x_{0}\right) \geqq f_{x_{0}}^{\prime} \eta, \quad-g_{J_{0}}\left(x_{0}\right) \geqq g_{x_{0}}^{\prime} \eta, \quad 0=h_{x_{0}}^{\prime} \eta .
$$

(b) If $x_{0}$ is a Kuhn-Tucker point then $x_{0}$ is a minimiser of the (scalar) optimisation problem (VOP).

PROOF. Since $f$ is a real-valued function an efficient point is exactly a minimiser. Therefore our corollary is a direct consequence of Theorem 4.1' and Proposition 4.1. (Observe that for a $C^{1}$-function the Fréchet derivative coincides with the Clarke subdifferential and that $g_{J_{0}}\left(x_{0}\right)=0$.)

REMARK 4.3. Corollary 4.1 is a generalisation of a result of Martin [13, Theorem 2.1] to programs with mixed constraints. In [13] Martin restricts himself to inequality constraints only.

Clearly, if $h$ is of class $C^{1}$ then KT-pseudoinvex-infine $\Rightarrow$ GKT-pseudo-invex-infine, KT-invex-infine $\Rightarrow$ GKT-invex-infine and HC-invex-infine $\Rightarrow$ GHC-invex-infine. We have seen from Theorem 4.1 that under suitable assumptions KT-pseudoinvex-infine $\Leftrightarrow$ GKT-pseudoinvex-infine. It is then natural to ask under which conditions we have

$$
\begin{aligned}
\text { KT-invex-infine } \Leftrightarrow \text { GKT-invex-infine, } \\
\text { HC-invex-infine } \Leftrightarrow \text { GHC-invex-infine. }
\end{aligned}
$$

The remainder of this paper is devoted to giving an answer to this question. Our results (Theorems 4.2 and 4.3) are also interesting from the point of view of sufficiency conditions for the efficiency property. Indeed, from the implication (b) $\Rightarrow$ (c) of Theorem 4.1 and the first part of Proposition 4.1 it is clear that conditions equivalent to GHC-invex-infineness or GKT-invex-infineness are sufficient conditions for a KuhnTucker point to be an efficient solution of $(V O P)$. The same is true for the case of HC-invex-infineness and KT-invex-infineness if $h$ is of class $C^{1}$ (see Theorem 4.1 and Proposition 4.1).

Let us introduce the following closedness conditions: 
$\left(\mathrm{H}_{2}\right)$ For any $i \in I$ and $x \in \bar{S}_{1}$ the set

$$
\begin{aligned}
\text { cone } & \left\{\bigcup_{i^{\prime} \neq i} \partial f_{i^{\prime}}\left(x_{0}\right) \times\left\{f_{i^{\prime}}\left(x_{0}\right)-f_{i^{\prime}}(x)\right\}, \bigcup_{j \in J_{0}} \partial g_{j}\left(x_{0}\right) \times\{0\}\right\} \\
& +\operatorname{sp}\left(\bigcup_{s \in L} \partial h_{s}\left(x_{0}\right) \times\{0\}\right)+\left[N_{S}\left(x_{0}\right) \times\{0\}\right]
\end{aligned}
$$

is closed.

$\left(\mathbf{H}_{2}\right)^{\prime}$ For any $i \in I, x \in \bar{S}_{1}$ and $u_{i^{\prime}}, v_{j}, w_{s}$ satisfying (4.6) the set

$$
\begin{aligned}
\text { cone } & \left\{\bigcup_{i^{\prime} \neq i} u_{i^{\prime}} \times\left\{f_{i^{\prime}}\left(x_{0}\right)-f_{i^{\prime}}(x)\right\}, \bigcup_{j \in J_{0}} v_{j} \times\{0\}\right\} \\
& +\operatorname{sp}\left(\bigcup_{s \in L} w_{s} \times\{0\}\right)+\left[N_{S}\left(x_{0}\right) \times\{0\}\right]
\end{aligned}
$$

is closed.

THEOREM 4.2. Consider the following statements:

(a) Problem (VOP) is KT-invex-infine at $x_{0} \in S_{1}$.

(b) Problem (VOP) is GKT-invex-infine at $x_{0} \in S_{1}$.

(c) If $\left(\lambda_{i}\right)_{i \in I} \geqq 0,\left(\mu_{j}\right)_{j \in J_{0}} \geqq 0,\left(r_{s}\right)_{s \in L} \in \mathbb{R}^{l}$ are such that (4.1) is satisfied then, for all $x \in \bar{S}_{1}, \sum_{i \in I} \lambda_{i} f_{i}(x) \geqq \sum_{i \in I} \lambda_{i} f_{i}\left(x_{0}\right)$ and, in addition, this inequality is strict in the case when $\left(\lambda_{i}\right)_{i \in I}>0$.

Then

(1) (a) $\Rightarrow$ (b) if h is of class $C^{1}$; and (b) $\Rightarrow$ (a) if h is of class $C^{1}$ and $\left(\mathrm{H}_{2}\right)$ holds.

(2) (b) $\Rightarrow$ (c); and (c) $\Rightarrow$ (b) if $\left(\mathrm{H}_{2}\right)^{\prime}$ holds.

(3) (a) $\Rightarrow$ (c) if $h$ is of class $C^{1}$; and (c) $\Rightarrow$ (a) if $h$ is of class $C^{1}$ and $\left(\mathrm{H}_{2}\right)$ holds.

ProOF. (a) $\Rightarrow$ (b) (if $h$ is of class $C^{1}$ ): Obviously.

(b) $\Rightarrow$ (a) (if $h$ is of class $C^{1}$ and $\left(\mathrm{H}_{2}\right)$ holds): This is a direct consequence of Definitions 4.2, 4.2' and Corollary 3.3 where $b_{i}=f_{i}(x)-f_{i}\left(x_{0}\right), c_{j}=0, a_{s}=0 ; S$ is replaced by $N_{S}\left(x_{0}\right)$; and $f_{i}, g_{j}$ and $h_{s}$ are replaced by $\overline{f_{i}}, \bar{g}_{j}$ and $\bar{h}_{s}$ (see (4.7)).

(b) $\Rightarrow$ (c): Let $\left(\lambda_{i}\right)_{i \in l} \geqq 0 ;\left(\mu_{j}\right)_{j \in J_{0}} \geqq 0,\left(r_{s}\right)_{s \in L} \in \mathbb{R}^{l}, u_{i} \in \partial f_{i}\left(x_{0}\right), v_{j} \in \partial g_{j}\left(x_{0}\right)$, $w_{s} \in \partial h_{s}\left(x_{0}\right)$ and $y \in N_{s}\left(x_{0}\right)$ be such that (4.8) holds. Then using (4.8) and Definition $4.2^{\prime}$, for any $x \in \bar{S}_{1}$ we find $\eta \in T_{S}\left(x_{0}\right)$ such that

$$
\sum_{i \in I} \lambda_{i}\left[f_{i}(x)-f_{i}\left(x_{0}\right)\right] \geqq\langle\zeta, \eta\rangle=\langle-y, \eta\rangle \geqq 0 .
$$

Thus $\sum_{i \in I} \lambda_{i}\left[f_{i}(x)-f_{i}\left(x_{0}\right)\right] \geqq 0$ and this inequality is strict if $\left(\lambda_{i}\right)_{i \in I}>0$. 
(c) $\Rightarrow$ (b) (if $\left(\mathrm{H}_{2}\right)^{\prime}$ holds): Assume to the contrary that for $x \in \bar{S}_{1}$ and $u_{i}, v_{j}, w_{s}$ satisfying (4.5) the system

$$
f(x)-f\left(x_{0}\right) \geq\langle u, \eta\rangle, \quad 0 \geqq\langle v, \eta\rangle, \quad 0=\langle w, \eta\rangle, \quad \eta \in T_{S}\left(x_{0}\right)
$$

has no solution. Then by Theorem 3.1 either the system

$$
\left\{\begin{array}{l}
\left(\lambda_{i}\right)_{i \in I}>0, \quad\left(\mu_{j}\right)_{j \in J_{0}} \geqq 0, \quad\left(r_{s}\right)_{s \in L} \in \mathbb{R}^{l}, \\
0 \in \sum_{i \in I} \lambda_{i} u_{i}+\sum_{j \in J_{0}} \mu_{j} v_{j}+\sum_{s \in L} r_{s} w_{s}+N_{S}\left(x_{0}\right), \\
\sum_{i \in I} \lambda_{i}\left[f_{i}\left(x_{0}\right)-f_{i}(x)\right]=0
\end{array}\right.
$$

or the system

$$
\left\{\begin{array}{l}
\left(\lambda_{i}\right)_{i \in I} \geqq 0, \quad\left(\mu_{j}\right)_{j \in J_{0}} \geqq 0, \quad\left(r_{s}\right)_{s \in L} \in \mathbb{R}^{l}, \\
0 \in \sum_{i \in I} \lambda_{i} u_{i}+\sum_{j \in J_{0}} \mu_{j} v_{j}+\sum_{s \in L} r_{s} w_{s}+N_{S}\left(x_{0}\right), \\
\sum_{i \in I} \lambda_{i}\left[f_{i}\left(x_{0}\right)-f_{i}(x)\right]>0
\end{array}\right.
$$

has a solution. This contradicts statement (c).

(a) $\Rightarrow$ (c) (if $h$ is of class $C^{1}$ ): This implication is obvious since (a) $\Rightarrow$ (b) and (b) $\Rightarrow$ (c).

(c) $\Rightarrow$ (a) (if $h$ is of class $C^{1}$ and if $\left(\mathrm{H}_{2}\right)$ holds): Assume to the contrary that for $x \in \bar{S}_{1}$ the system

$$
f(x)-f\left(x_{0}\right) \geq \bar{f}(\eta), \quad 0 \geqq \bar{g}_{J_{0}}(\eta), \quad 0=\bar{h}(\eta), \quad \eta \in T_{S}\left(x_{0}\right),
$$

has no solution where $\bar{f}, \bar{g}_{J_{0}}$ and $\bar{h}$ are defined by (4.7). Then by Theorem 3.1 either the system (4.18)-(4.20) or the system (4.21)-(4.23) has a solution where $w_{s}=h_{s x_{0}}^{\prime}$; and $u_{i} \in \partial f_{i}\left(x_{0}\right)$ and $v_{j} \in \partial g_{j}\left(x_{0}\right)$ are suitable points. (Observe that the points $u_{i}$ and $v_{j}$ which appear in (4.19) and (4.22) may be different points.) This contradicts statement (c).

We conclude our paper by formulating Theorem 4.3 whose proof is similar to that of Theorem 4.2 and is omitted. For this purpose, let us introduce the following closedness conditions:

$\left(\mathrm{H}_{3}\right)$ For any $i \in I$ and $x \in \bar{S}_{1}$ the set

$$
\begin{aligned}
\text { cone } & \left\{\bigcup_{i^{\prime} \neq i} \partial f_{i^{\prime}}\left(x_{0}\right) \times\left\{f_{i^{\prime}}\left(x_{0}\right)-f_{i^{\prime}}(x)\right\}, \bigcup_{j \in J_{0}} \partial g_{j}\left(x_{0}\right) \times\left\{g_{j}\left(x_{0}\right)-g_{j}(x)\right\}\right\} \\
& +\operatorname{sp}\left(\bigcup_{s \in L} \partial h_{s}\left(x_{0}\right) \times\{0\}\right)+\left[N_{S}\left(x_{0}\right) \times\{0\}\right]
\end{aligned}
$$


is closed.

$\left(\mathrm{H}_{3}\right)^{\prime} \quad$ For any $i \in I, x \in \bar{S}_{1}$ and $u_{i^{\prime}}, v_{j}, w_{s}$ satisfying (4.6) the set

$$
\begin{aligned}
\text { cone } & \left\{\bigcup_{i^{\prime} \neq i} u_{i^{\prime}} \times\left\{f_{i^{\prime}}\left(x_{0}\right)-f_{i^{\prime}}(x)\right\}, \bigcup_{j \in J_{0}} v_{j} \times\left\{g_{j}\left(x_{0}\right)-g_{j}(x)\right\}\right\} \\
& +\operatorname{sp}\left(\bigcup_{s \in L} \partial h_{s}\left(x_{0}\right) \times\{0\}\right)+\left[N_{S}\left(x_{0}\right) \times\{0\}\right]
\end{aligned}
$$

is closed.

THEOREM 4.3. Consider the following statements:

(a) Problem (VOP) is $H C$-invex-infine at $x_{0} \in S_{1}$.

(b) Problem (VOP) is GHC-invex-infine at $x_{0} \in S_{1}$.

(c) If $\left(\lambda_{i}\right)_{i \in I} \geqq 0,\left(\mu_{j}\right)_{j \in J_{0}} \geqq 0,\left(r_{s}\right)_{s \in L} \in \mathbb{R}^{l}$ are such that $(4.1)$ is satisfied then for any $x \in \bar{S}_{1}, \zeta(x) \geqq \zeta\left(x_{0}\right)$ and, in addition, this inequality is strict in the case $\left(\lambda_{i}\right)_{i \in I}>0$ where

$$
\zeta(x)=\sum_{i \in I} \lambda_{i} f_{i}(x)+\sum_{j \in J_{0}} \mu_{j} g_{j}(x)
$$

Then

(1) (a) $\Rightarrow$ (b) if $h$ is of class $C^{1}$; and (b) $\Rightarrow$ (a) if h is of class $C^{1}$ and $\left(\mathrm{H}_{3}\right)$ holds.

(2) (b) $\Rightarrow$ (c); and (c) $\Rightarrow$ (b) if $\left(\mathrm{H}_{3}\right)^{\prime}$ holds.

(3) (a) $\Rightarrow$ (c) if $h$ is of class $C^{1}$; and (c) $\Rightarrow$ (a) if $h$ is of class $C^{1}$ and $\left(\mathrm{H}_{3}\right)$ holds.

REMARK 4.4. Let us observe that the convex cone generated by a finite number of points is always closed. Hence the closedness assumptions $\left(\mathrm{H}_{1}\right)^{\prime},\left(\mathrm{H}_{2}\right)^{\prime}$ and $\left(\mathrm{H}_{3}\right)^{\prime}$ are automatically satisfied for the case when $S=X$ and the equality constraints are absent. This remark is useful since many practical problems involve only inequality constraints and hence for such problems we do not need to check these assumptions.

\section{Acknowledgement}

This work was supported by Korea Research Foundation under Grant KRF-99-042D00018.

\section{References}

[1] J. P. Aubin, Mathematical methods of game and economic theory (North-Holland, Amsterdam, 1979). 
[2] H. P. Benson, "Concave minimization: theory, applications and algorithms", in Handbook of global optimization (eds. R. Horst and P. M. Pardalos), (Kluwer, Dordrecht, 1995) 43-148.

[3] D. Blackwell and M. A. Girshick, Theory of games and statistical decisions (John Wiley and Sons, New York, 1954).

[4] A. J. V. Brandas, M. A. Rojas-Medar and G. N. Silva, "Invex nonsmooth alternative theorem and applications", Optimization 48 (2000) 239-253.

[5] F. H. Clarke, Optimization and nonsmooth analysis (Wiley-Interscience, New York, 1983).

[6] B. D. Craven, "Nondifferentiable optimization by nonsmooth approximations", Optimization 17 (1986) 3-17.

[7] T. S. Ferguson, Mathematical statistics: a decision theoretic approach (Academic Press, New York, 1967).

[8] A. M. Geoffrion, "Proper efficiency and the theory of vector maximization", J. Math. Anal. Appl. 22 (1968) 618-630.

[9] M. A. Hanson, "On sufficiency of the Kuhn-Tucker conditions", J. Math. Anal. Appl. 80 (1981) 545-550.

[10] M. A. Hanson, "Invexity and the Kuhn-Tucker theorem", J. Math:Anal. Appl. 236 (1999) 594-604.

[11] M. A. Hanson and B. Mond, "Necessary and sufficient conditions in constrained optimization", Math. Programming 37 (1987) 51-58.

[12] O. L. Mangasarian, Nonlinear programming (McGraw-Hill, New York, 1969).

[13] D. H. Martin, "The essence of invexity", J. Optim. Th. Appl. 47 (1985) 65-76.

[14] R. Osuna-Gómez, A. Beato-Moreno and A. Rufian-Lizana, "Generalized convexity in multiobjective programming", J. Math. Anal. Appl. 233 (1999) 205-220.

[15] R. Osuna-Gómez, A. Rufian-Lizana and P. Ruiz-Canalez, "Invex functions and generalized convexity in multiobjective programming", J. Optim. Th. Appl. 98 (1998) 651-661.

[16] V. Pareto, Manuale di economia politica, con una introduzione ulla scienza sociale (Societa Editrice Libraria, Milan, Italy, 1906).

[17] T. D. Phuong, P. H. Sach and N. D. Yen, "Strict lower semicontinuity of the level sets and invexity of a locally Lipschitz function", J. Optim. Th. Appl. 87 (1995) 579-594.

[18] T. W. Reiland, “Nonsmooth invexity", Bull. Austral. Math. Soc. 42 (1990) 437-446.

[19] P. H. Sach, D. S. Kim and G. M. Lee, "Generalized convexity and nonsmooth problems of vector optimization", Preprint 2000/31, Hanoi Institute of Mathematics, (submitted).

[20] P. H. Sach, D. S. Kim and G. M. Lee, "Invexity as necessary optimality condition in nonsmooth programs", Preprint 2000/30, Hanoi Institute of Mathematics, (submitted).

[21] P. H. Sach, G. M. Lee and D.S. Kim, "Infine functions, nonsmooth alternative theorems and vector optimization problems", J. Global Optim. 27 (2003) 51-81.

[22] A. Takayama, Mathematical economics (The Dryden Press, Illinois, 1974).

[23] Z. Xu, "Generalization of nonhomogeneous Farkas' lemma and applications", J. Math. Anal. Appl. 186 (1994) 726-734.

[24] M. Zeleny, Multiple criteria decision making (McGraw-Hill, New York, 1982). 